\title{
Division of Labor as the Result of Phase Transition
}

\author{
Jinshan $\mathrm{Wu}^{1,2 *}$, Zengru $\mathrm{Di}^{1}$, Zhanru Yang ${ }^{1,2}$ \\ 1. Department of Physics, Beijing Normal University, Beijing, P.R.China \\ 2. Institute of Theocratical Physics, Beijing Normal University, Beijing, P.R.China
}

November 29, 2018

\begin{abstract}
The emergence of labor division in multi-agent system is analyzed by the method of statistical physics. Considering a system consists of $\mathrm{N}$ homogeneous agents. Their behaviors are determined by the returns from their production. Using the Metropolis method in statistical physics, which in this model can been regarded as a kind of uncertainty in decision making, we constructed a Master equation model to describe the evolution of the agents distribution. When we introduce the mechanism of learning by doing to describe the effect of technical progress and a formula for the competitive cooperation, the model gives us the following interesting results: (1) As the results of long term evolution, the system can reach a steady state. (2) When the parameters exceed a critical point, the labor division emerges as the result of phase transition. (3) Although the technical progress decides whether or not phase transition occurs, the critical point is strongly effected by the competitive cooperation. From the above physical model and the corresponding results, we can get a more deeply understanding about the labor division.
\end{abstract}

Key Words: Division of Labor, Statistical Physics, Phase Transition, Metropolis Method

Pacs:05.45.-a; 87.23-n

\section{Introduction}

The economic system is no doubt a many-particle system-it can be viewed as a collection of numerous interaction agents. So it is possible that methods and concepts developed in the study of strongly fluctuation systems might yield new results in this area. In fact, in the past decades the approaches from statistical physics have been applied in economics and a lot of interesting results, including empirical laws and theoretical

*Electrical address: wujinshan@yahoo.com 
models, have been achieved (see [1, 2, 3, 4] and [5] as a review of Econophysics). Among all these studies, a great deal of researchers is on the agent-based modelling and related non-trivial self-organizing phenomena. In the economic system, the agents learn from each other, and their activities may be influenced by others' actions. These interactions between agents may be simple and local, but they may have important consequence related with the emergence of global structure. To understand the mechanism behind these innovation phenomena, the methods and concepts in phase transitions and critical phenomena are helpful. For instance, in the study of majority and minority game 6, 7, 8], opinion formation [9], and computational ecosystems [10].

In this paper, we focus on the formation of labor division. Roughly speaking, an economic organizational pattern is said to involve the division of labor if it allocates the labor of different individuals to different activities. Hence the specialization of individuals and the number of professional activities are two sides of division of labor [11]. It is a common functional organization observed in many complex systems, and it is a fundamental way to improve efficiency and utilization so as to get global optimization for the system. In order to investigate the mechanism behind the formation of labor division, we have constructed a simple model with many interacting agents. Every agent has only two kind of tasks, namely $A$ and $B$. we describe the level of specialization of agent by his working-time share spent on producing $A$ or $B$. Each agent make their decisions for working-time in different tasks, and receive payoffs according to their and other agents' choices. The agents can adapt by evaluating the performance of their strategies from past experience so as to get maximum returns. The returns for any agent is determined by its production with endogenous technical progress-through the mechanism of learning by doing, and its cooperation with other agents. Just like the Hamiltonian in statistical physics, the payoff function determines the behavior of the agent in economic system. Because of the bounded rationality and incompleteness information in the system, we have introduced a parameter, named social temperature $T$ (in the model, we have absorbed the $\beta=k_{B} T$ into the other parameters. Such an approach is traditionally used in Statistical Physics, for example, let $H^{\prime}=\beta H$, so the new $J$ in Hamiltonian of Ising model means $\beta J$ actually.), to describe the degree of randomness in decision-making. Then we assume that the system should obey the canonical ensemble distribution, that is the probability $P(\vec{x})$ of a microstate $\vec{x}$ is proportional to its 'Boltzmann factor' determined by the total returns of the microstate $\vec{x}$ :

$$
P(\vec{x})=\frac{e^{+\beta E(\vec{x})}}{\int e^{+\beta E(\vec{x})} d \vec{x}}
$$

Then using the Metropolis simulation method [12, 13], we can get a Master equation to investigate the evolution of the system. With the continual change of system parameters, we have found a so called 'social phase transitions' phenomenon related to the emergence of labor division.

The model is defined in Section 2. The economies of specialization is introduced by increasing returns from learning by doing. And the economies of complementarity 
is described by an additional payoffs from the combination of two products. Section 3 gives the numerical results. The effects of parameters on critical point are discussed in detail. It is revealed that although the technical progress is a key factor that determine whether or not the labor division will happen, the competitive cooperation among agents has very important effects on the critical point. Our results are summarized in Section 4.

\section{The Model}

Let's consider a system consists of $N$ homogeneous agents. In a given time period $T$, any agent has two different kind of tasks, namely $A$ and $B$. In any time unit, the fraction of working time on task $A$ for ith agent is denoted by $p^{i}(t) \in[0,1]$. we are interested in the long-term $(T \rightarrow \infty)$ evolution of the system, especially the emerging of labor division.

$p^{i}$ is a real number in $[0,1]$, which describes the working pattern for the ith agent. If $p^{i}$ equals 1 or 0 , the agent is full specialized in $A$ or $B$ and we call the system is in complete division of labor. If $p^{i}$ equals 0.5 , it means that the agent spends same time interval on tasks $A$ and $B$, and we call it as the time-dividing working mode in the following discussion. Usually $p^{i}$ could be any real number between 0 and 1 , in this case the agent does not have any preference on job $A$ or $B$. We focus on the global behavior of the system. Based on the above description of the agent, we introduce the following two order parameters to describe the behavior of the system on macroscopic level:

$$
\begin{aligned}
& \lambda_{1}(t)=\frac{1}{N} \sum_{i}\left|2 p^{i}(t)-1\right| \\
& \lambda_{2}(t)=\frac{1}{N} \sum_{i}\left(2 p^{i}(t)-1\right)
\end{aligned}
$$

Where $\lambda_{1}$ describes the intensity of labor division and cooperation in the system. It has three special values, $\{0,0.5,1\}$, represent time-dividing working mode, every $p^{i}$ equals 0.5 , no-preference working mode, $p^{i}$ distributed in $(0,1)$ randomly, and full specialization, every $p^{i}$ equals 0 or 1 , respectively. $\lambda_{2}$ gives the agents allocation on tasks $A$ and $B$ on global (in average on macroscopic level).

In the following discussion, we try to specify a suitable evolution rule for $p^{i}(t)$ based on the similar approach in statistical physics. So we can then determine the dynamical equations for $p^{i}(t)$, and get the final steady states for $\lambda_{1}(t)$ and $\lambda_{2}(t)$.

Analogous with Ising model in statistical physics, $p^{i}$ could be treated as the spin in ith point of the lattice, and then $\lambda_{2}$ is the magnetization. So if we can give the Hamiltonian of the system, the evolution of the system could be determined. In the economic system, the agent switches his behavior according to his evaluations on the returns from production. Every agent try to get maximum return just as any practical

tends to stay in the state with lowest energy. So the payoff function from the agent's 
production should have the same effect as the Hamiltonian. The working mode for each agent should be determined by its returns.

\subsection{Production function}

According to the previous literatures on specialization and economic organization, we know that there are several factors that related to the division of labor, such as the increasing returns to specialization and transaction costs. In fact, limited ability on learning and incomplete information on the technology will lead to the labor division directly. But we don't take them into account in this model. In our model, every agent know the technology for producing $A$ and $B$. And for any agent there is not any comparative advantages for producing $A$ or $B$ in the initial. The technical progress is achieved by the mechanism of learning by doing without any cost. The payoff function for agent $i$ is given by the following formulas:

$$
\varepsilon^{i}=\varepsilon_{\text {tech }}^{i}+\varepsilon_{\text {self }}^{i}+\varepsilon_{c o}^{i}
$$

where

$$
\begin{gathered}
\varepsilon_{\text {tech }}^{i}=p^{i} \times \Gamma_{A}^{i}+\alpha\left(1-p^{i}\right) \times \Gamma_{B}^{i} \\
\varepsilon_{\text {self }}^{i}=\beta \min \left(p^{i} \times \Gamma_{A}^{i},\left(1-p^{i}\right) \times \Gamma_{B}^{i}\right) \\
\varepsilon_{c o}^{i}=\frac{1}{2} \beta\left(\Gamma_{B}^{i}\left(1-p^{i}\right)-\Gamma_{A}^{i} p^{i}\right) \sum_{j \neq i}\left(\Gamma_{A}^{j} p^{j}-\Gamma_{B}^{j}\left(1-p^{j}\right)\right)
\end{gathered}
$$

In the functions above, $\Gamma_{A}^{i}$ and $\Gamma_{B}^{i}$ are the technology for producing $A$ and $B$ respectively. For simplicity and without losing any generality, we assume that unit production $A$ will get unit return. And $\alpha \in(1, \infty)$ is the return of unit production $B$. $\varepsilon_{\text {self }}^{i}$ gives the additional benefits for composite $A$ and $B$ into a final product. So it is related to the less one between $A$ and $B$. If agent $i$ has some more single product after the composition himself, he can also get another return, named $\varepsilon_{c o}^{i}$, from the cooperation with other agents. But the return got from the composition with the product from other agent is factored by $\frac{1}{2} \beta$. Because of the incomplete information in the system, any agent couldn't know the situation of production on global. So in order to get the corresponding returns $\varepsilon_{c o}^{i}$ for every agent, we used the average-field approach similar with that in statistical physics. That means, for every agent, he will match his product with all the other agents and then the result is the average. This assumption indicate that there are already a public market in economics. In the function for $\varepsilon_{c o}^{i},\left(\Gamma_{B}^{i}\left(1-p^{i}\right)-\Gamma_{A}^{i} p^{i}\right)$ gives the surplus product $B$ for agent $i$, and the sum of $\left(\Gamma_{A}^{j} p^{j}-\Gamma_{B}^{j}\left(1-p^{j}\right)\right)$ gives the surplus product $A$ of the all other agents. When these two terms have different signs, their product is negative and $\varepsilon_{c o}^{i}$, the corresponding payoff from the cooperation, will be also negative. In this case, the surplus product of the agent is the same kind as the final surplus of all the other agents. Because of the transaction cost and the diseconomies of incomplementarity, it is rationale that the agent would get negative payoff. 


\subsection{Two descriptions for learning by doing}

The way for technical progress is learning by doing. This mechanism is related to the accumulation of agent's historical production behavior. That means the development of comparative advantages is determined by related working time. Let $x_{\text {Aor } B}^{i}$ denote the integral working time on $A$ or $B$ for agent $i$ :

$$
\begin{gathered}
x_{A}^{i}=\int_{0}^{t} d \tau p^{i}(\tau) \\
x_{B}^{i}=\int_{0}^{t} d \tau\left(1-p^{i}(\tau)\right)
\end{gathered}
$$

We have introduced two mechanisms for technical progress, named $\gamma$-mechanism and $\mu$-mechanism. They are given by following functions:

$$
\begin{gathered}
\Gamma_{A / B}^{i}=\gamma^{x_{A / B}^{i}} \\
\Gamma_{A / B}^{i}=1+\frac{\mu x_{A / B}^{i}{ }^{2}}{\nu+x_{A / B}^{i}{ }^{2}}
\end{gathered}
$$

These two mechanisms will give different results. Equation(9) is an exponential function which gives unlimited growth on technology, while there is an upper limit in equation(10). Figure(11) shows three functions for $\Gamma_{A / B}^{i}$ in our computer simulations.

\subsection{The Master equation for the system evolution}

In the production function discussed above, term $\varepsilon_{c o}^{i}$ is the return from the agents' cooperation. It introduces interactions among all agents (the same as the interactions among spins). So if we discuss the dynamics in $\mu$-space, we should construct $N$ evolutionary equations for $p^{i}$. But they are difficult for both theoretical analysis and

computer simulation. In the following discussion, we try to describe the evolution of the system in $\Gamma$-space. That is to discuss the evolutionary behavior of the joint density function $P\left(\left\{p^{i}\right\}, t\right)$ in the space sponsored by $\left\{p^{i}\right\}$. Then the total production for the system is:

$$
E=\sum_{i=1}^{N} \varepsilon_{i}
$$

Where $\varepsilon_{i}$ is the production of agent $i$ given by eq(41). The same as the approach of critical dynamics in Ising model, the Master equation for the joint density function $P\left(\left\{p^{i}\right\}, t\right)$ in $\Gamma$ space is

$$
\frac{d}{d t} P\left(p^{1}, \ldots, p^{i}, \ldots, p^{N} ; t\right)=\sum_{i} \int_{0}^{1} d x^{i} \begin{gathered}
{\left[\omega^{i}\left(x^{i} \rightarrow p^{i}\right) P\left(p^{1}, \ldots, x^{i}, \ldots, p^{N} ; t\right)\right.} \\
\left.-\omega^{i}\left(p^{i} \rightarrow x^{i}\right) P\left(p^{1}, \ldots, p^{i}, \ldots, p^{N} ; t\right)\right]
\end{gathered}
$$




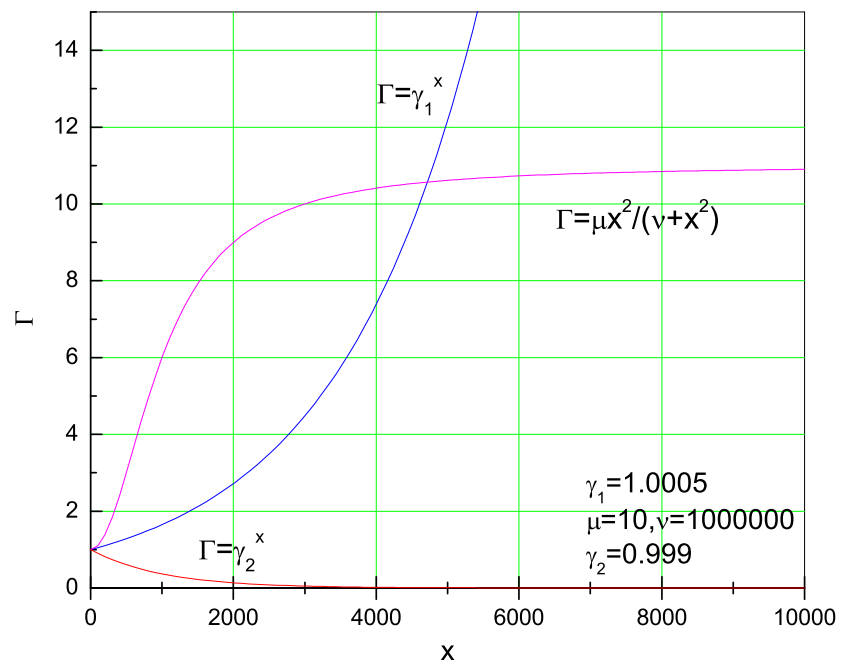

Figure 1: Sample functions of $\Gamma^{i}$ used in the simulations. Parameters are labelled in the graph.

where $\omega^{i}$ is the transition probability which is determined by the Boltzman factors as the following:

$$
\omega^{i}\left(p^{i} \rightarrow x^{i}\right)=\frac{e^{\Delta E\left(p^{i} \rightarrow x^{i}\right)}}{\int_{0}^{1} d x^{i} e^{\Delta E\left(p^{i} \rightarrow x^{i}\right)}}
$$

In the above transition probabilities, $\Delta E\left(p^{i} \rightarrow x^{i}\right)$ is the change of returns related to the variation of working mode for $i t h$ agent. From eq.(11) we can get

$$
\begin{gathered}
{\left[\left(\Gamma_{A}^{i}-\alpha \Gamma_{B}^{i}+\frac{1}{2} \beta\left(\Gamma_{A}^{i}-\Gamma_{B}^{i}\right)\right)\left(x^{i}-p^{i}\right)\right.} \\
-\frac{\beta}{2}\left(\left|x^{i} \Gamma_{A}^{i}-\left(1-x^{i}\right) \Gamma_{B}^{i}\right|-\left|p^{i} \Gamma_{A}^{i}-\left(1-p^{i}\right) \Gamma_{B}^{i}\right|\right) \\
\left.+2 \varepsilon_{c o}^{i}\left(x^{i}\right)-2 \varepsilon_{c o}^{i}\left(p^{i}\right)\right]
\end{gathered}
$$

With the master equation(12) and certain initial conditions, we can get evolving behaviors of $\lambda_{1}$ and $\lambda_{2}$. Then we can discuss the phenomena on labor division. It is difficult to have some theoretical results. So in the next section we will show some numerical simulation results by Monte Carlo simulation method. Based on the Master equation(12) and corresponding transition probability(13), the simulation is proceed on the following Metropolis algorithm:

1. For any given state with $p^{i}$ for agent $i$, a new state $x^{i}$ is randomly selected;

2. If $\Delta E\left(p^{i} \rightarrow x^{i}\right)>0$, then the transition from $p^{i}$ to $x^{i}$ is proceeded; 


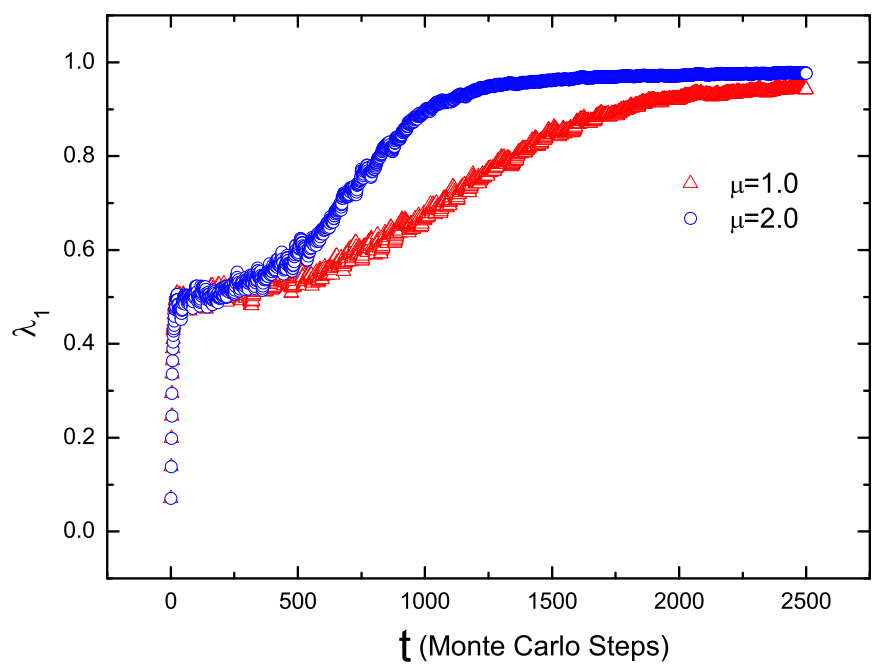

Figure 2: A typical evolutionary behavior of the model under $\mu$-mechanism. $\beta=10.0$, $\alpha=1.0$.

3. If $\Delta E\left(p^{i} \rightarrow x^{i}\right) \leq 0$, then a random number $\xi \in(0,1)$ is selected. If $\xi \leq e^{\beta \Delta E}$, the transition from $p^{i}$ to $x^{i}$ is proceeded or else the agent $i$ keeps the original state.

4. For another agent $j$, goto step 1.

For any given initial state, the system will achieve a certain steady state after some transient process. Figure(2) gives a typical evolution behavior under $\mu$ mechanism.

\section{The Results of Monte Carlo Simulation}

There are several parameters related to the mechanism of labor division in the model. We will show the simple or maybe trivial results of $\gamma$-mechanism first and then emphasize our discussion on the results of $\mu$-mechanism. And we let $\alpha=1$ in all the simulations before we discuss the effects of parameters $\alpha$ and $N$ on the system evolution in the end.

\section{$3.1 \gamma$-mechanism}

As shown in(3) because of $\gamma$-mechanism is described by an exponential function, the system will achieve the state of labor division for any given initial state and $\gamma>1$ 


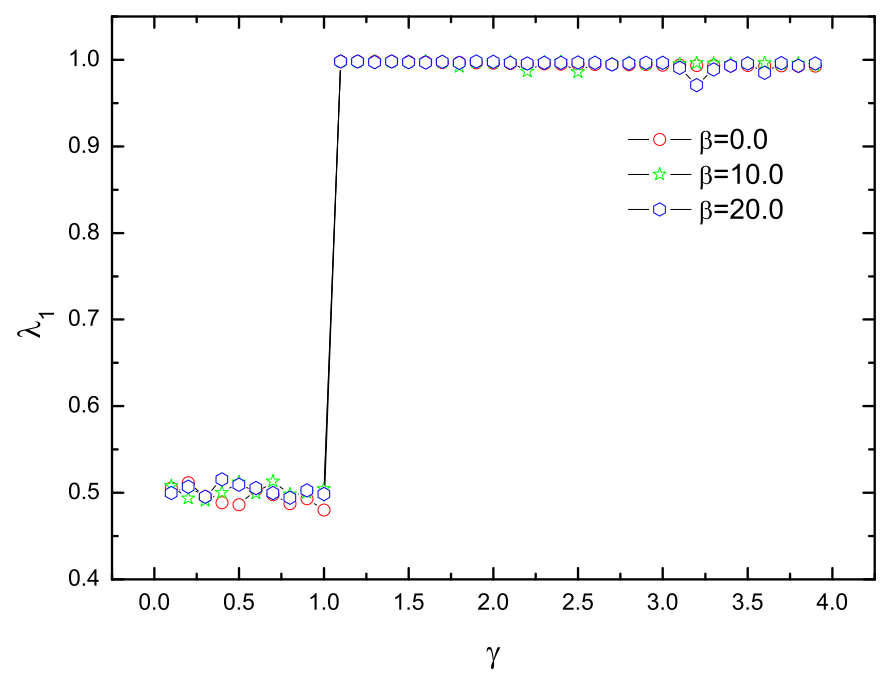

Figure 3: Parameter $\lambda_{1}$ in the final stationary state as a function of parameter $\gamma$ under $\gamma$-mechanism. The final steady state of the system is determined by $\gamma>1$ or $\gamma<1$.

no matter how small is $\gamma-1$. That means if the intensity of technical progress is big enough, the agent would definitely specialized in a kind of task. Other factors such as competitive cooperation described by parameter $\beta$ have no effects on the long term evolution.

\section{$3.2 \mu$-mechanism}

But for the model with $\mu$-mechanism, $\beta$ has some important effects on the system evolution. As shown in Figure(何), there are phase transitions results in labor division and $\beta$ has strong effects on the critical point $\mu_{c}$ for parameter $\mu . \mu_{c}$ decreases as the $\beta$ increasing. The $\mu$-mechanism for learning by doing gives a logistic growth for technology. That is much more realistic than the $\gamma$-mechanism. The results reveal that the competitive cooperation among agents is very important for the emergence of labor division. However, as discussed in the end of this section, when $\mu=0$, that is there is no mechanism of increasing returns, the system has no phase transition to labor division. This result is rationale for the model and it is consistent with the theory on specialization. The comparative advantages in production can only be introduced by a positive feedback caused by increasing returns. So the mechanism of learning by doing is a dominant factor for labor division.

In order to investigate the effects of all terms in production function in detail, we have simulated some special cases. The first case is $\beta=0$. Then $\varepsilon_{\text {self }}^{i}$ and $\varepsilon_{c o}^{i}$ in equation (四) are all zero and technical progress is the only one factor that affects the 


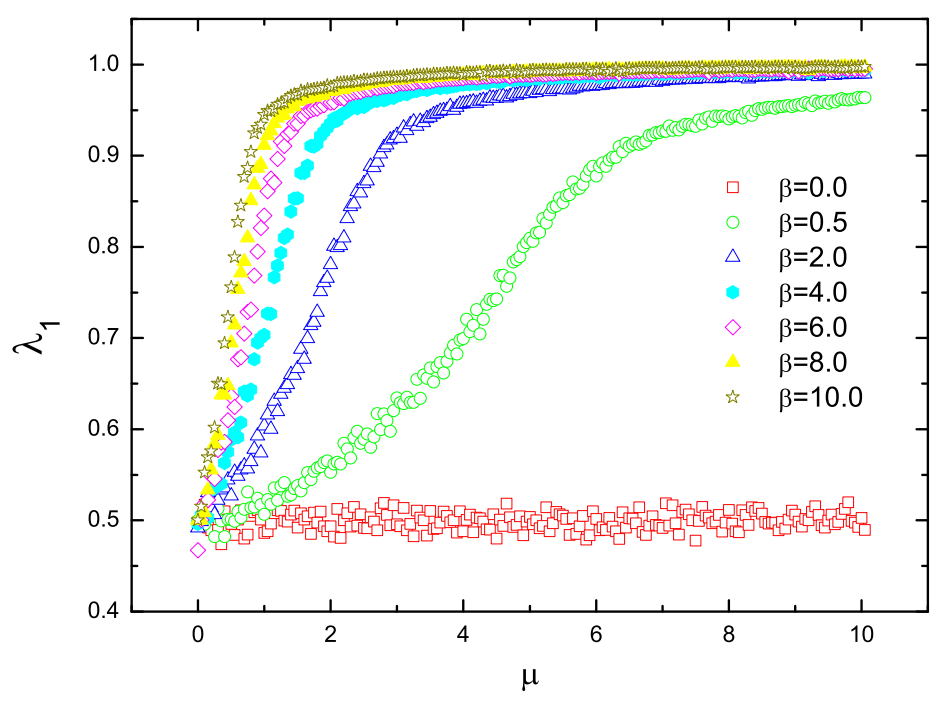

Figure 4: For different parameter $\beta$, parameter $\lambda_{1}$ in the final stationary state as a function of parameter $\mu$ under $\mu$-mechanism. There is phase transition in the system. $\beta$ has strongly effects on the critical point.

final results. As shown in figure(5), there is a phase transition in the system. Under the given conditions, the critical point for $\mu\left(\mu_{c}\right)$ is around 50 .

Then we have simulated the model with $\varepsilon_{\text {self }}^{i}$ but no $\varepsilon_{c o}^{i}$. The results are shown in (6). When $\beta$ is greater than a critical value $\beta_{c}$, the system will stabilized in the state with $\lambda_{1}=0$. That is $p^{i}=0.5$ for any agent $i$. Every agent does the tasks $A$ and $B$ himself and spends the same time on both tasks. The system is in the time-dividing working mode. Only when $\beta$ is smaller than $\beta_{c}$, does the system have phase transition to labor division. But the critical value $\mu_{c}$ is much larger than that of in Figure (目). It is even larger than the $\mu_{c}$ in Figure (5). So the term $\varepsilon_{\text {self }}^{i}$ has the effect of antispecialization. From the simulation results in Figure (4) and just as we have indicated in the beginning of this section, $\varepsilon_{c o}^{i}$ is helpful to labor division. Actually the term $\varepsilon_{c o}^{i}$ is the benefit for agent $i$ get from the cooperation with all the other agents. It should has the same effect as the decreasing of transaction costs. So it is not surprising that this term will enforce specialization.

Another interesting case is the system without technical progress, that is $\Gamma_{A}^{i}$ and $\Gamma_{B}^{i}$ are all equal 1. In this case, the system is exactly the same as Ising model. Let $S^{i}=2 p^{i}-1$ and $\alpha=1$, then the total returns for the system is

$$
E=-\beta \sum_{i, j, i \neq j} S^{i} S^{j}+\frac{1}{2} \sum_{i} \beta\left(1-\left|S^{i}\right|\right)
$$




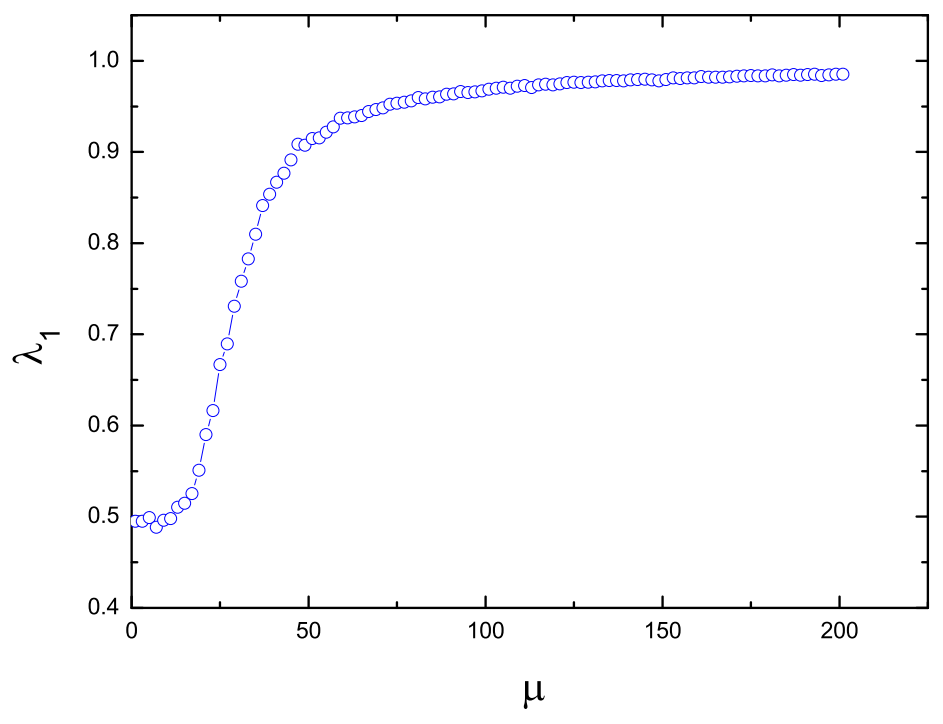

Figure 5: Under $\mu$-mechanism and without the term $\varepsilon_{c o}^{i}$ that is the payoff from the cooperation among agents, parameter $\lambda_{1}$ in the final stationary state as a function of parameter $\mu$ for different parameter $\beta$. The critical point $\mu_{c}$ is much larger than that in Fig. 4 and even in Fig. 6. 


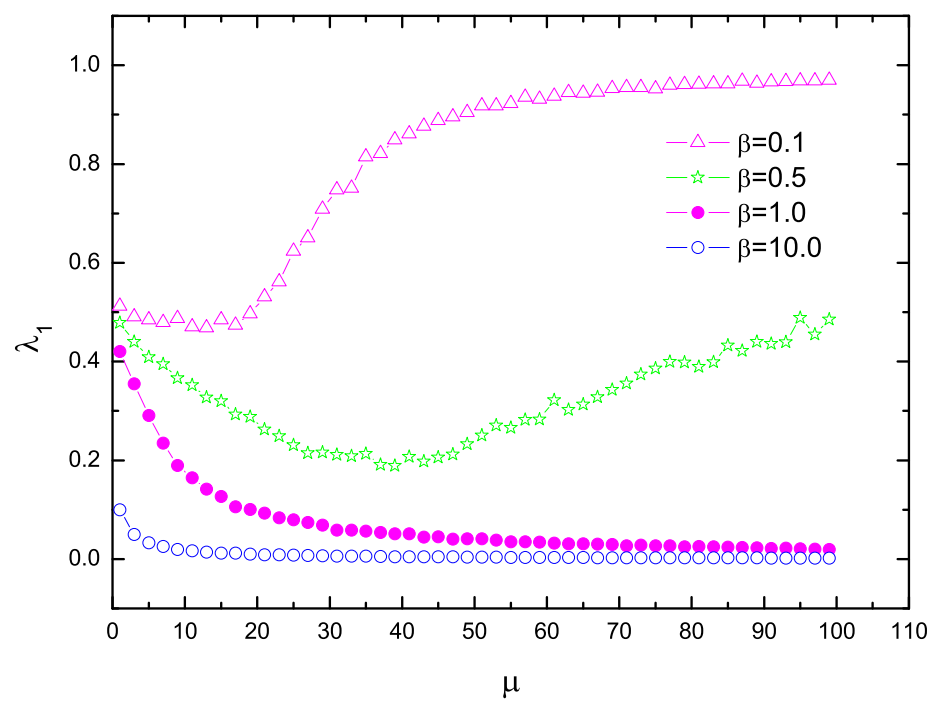

Figure 6: Under $\mu$-mechanism, parameter $\lambda_{1}$ in the final stationary state as a function of parameter $\mu$ when there is only the term for technical progress in the production function. The critical point $\mu_{c}$ is much larger than that in Fig. 4. 


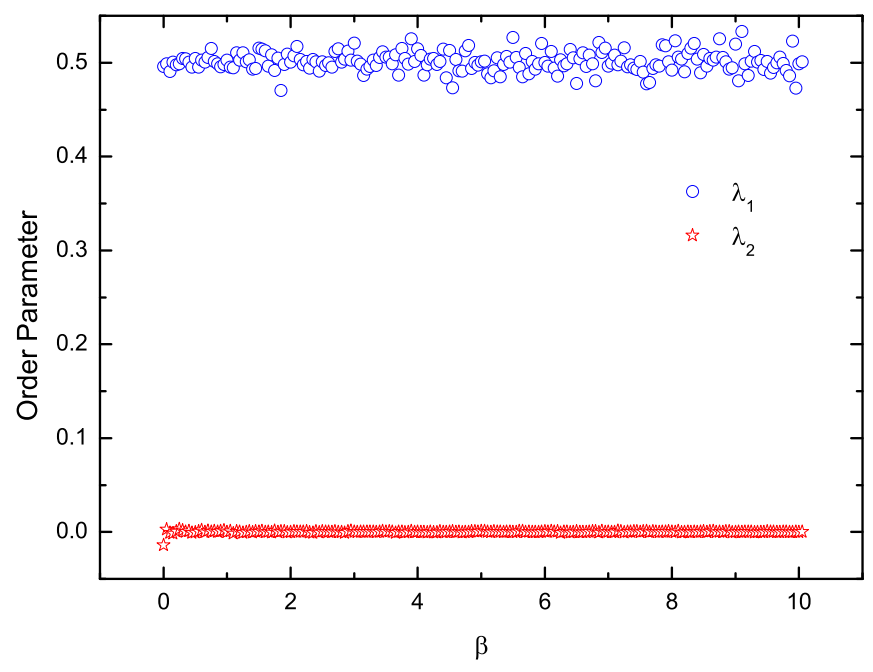

Figure 7: Order parameters $l a m b d a_{1}$ and $\lambda_{2}$ as a function of parameter $\beta$ when there is no progress of productivity. The system has no phase transition.

Where $S^{i} \in(-1,1)$. The distribution of the state for the system is determined by $e^{E}$. Comprising with Ising model, let the corresponding Hamiltonian is

$$
H=-\frac{1}{2} \sum_{i} \beta\left(1-\left|S^{i}\right|\right)+\beta \sum_{i \neq j} S^{i} S^{j}
$$

and let $k_{\beta} T=1$, then the system could be described by Canonical Ensemble with $e^{-\frac{H}{k_{\beta} T}}$. That is an anti-ferromagnetic Ising model with global interaction. The similar approach shows that this system either does't have phase transition related to labor division. The result is shown in Figure(7).

\subsection{The effects of parameter $\alpha$ and $N$}

Parameter $\lambda_{2}$ reflects the preference for tasks $A$ and $B$. So it is expected that parameter $\alpha$, the ratio of returns for $A$ and $B$, will affect final results on $\lambda_{2}$. The diagram in phase space for $\lambda_{2}$ when $\alpha=1$ is shown in Figure (8), in which we can see $\lambda_{2}$ fluctuates around zero, and no phase transition with symmetry breaking emerges. And as shown in Figure(9), $\alpha$ indeed has some effects on $\lambda_{2}$. But because of the combination mechanism described by parameter $\beta, \alpha$ has only a little effect on $\lambda_{2}$. It also has some detailed effects on labor division described by $\lambda_{1}$, as shown in Figure (10). But $\alpha$ could not change the qualitative behavior of phase transition.

Here are other two phenomena that should be pay attention to, first, the model has no scale effect for limited $N$, as shown in Figure (11). The phase curves for different $N$ 


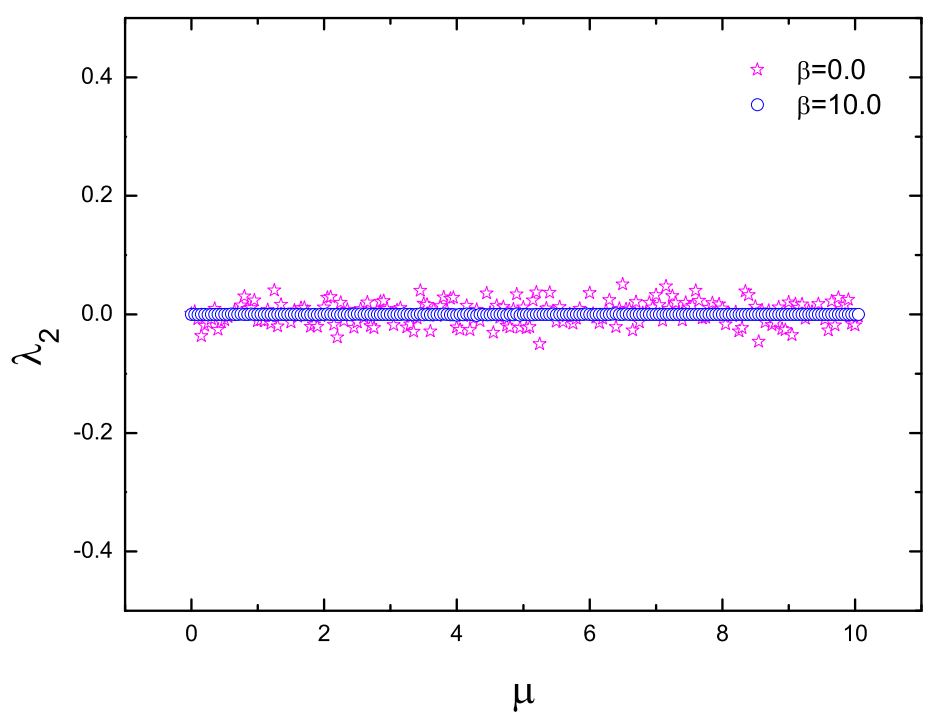

Figure 8: Parameter $\lambda_{2}$ in the final stationary state as a function of parameter $\mu$ for different $\beta$ when $\alpha=1$. There is no symmetry breaking with the phase transition.

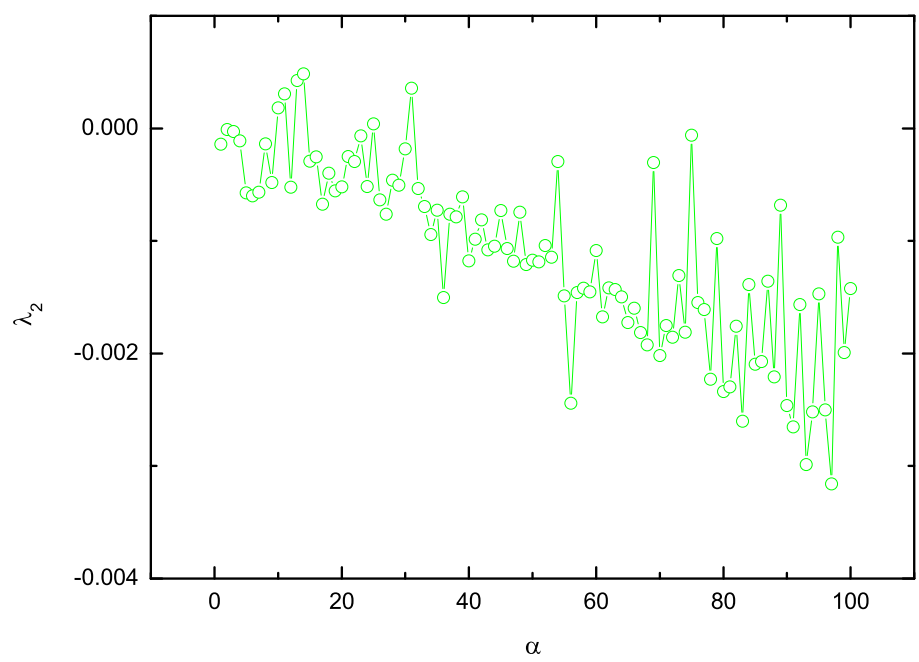

Figure 9: Parameter $\lambda_{2}$ in the final stationary state as a function of parameter $\alpha$. Because of the cooperation mechanism described by parameter $\beta, \alpha$ has only a little effect on the final distribution. 


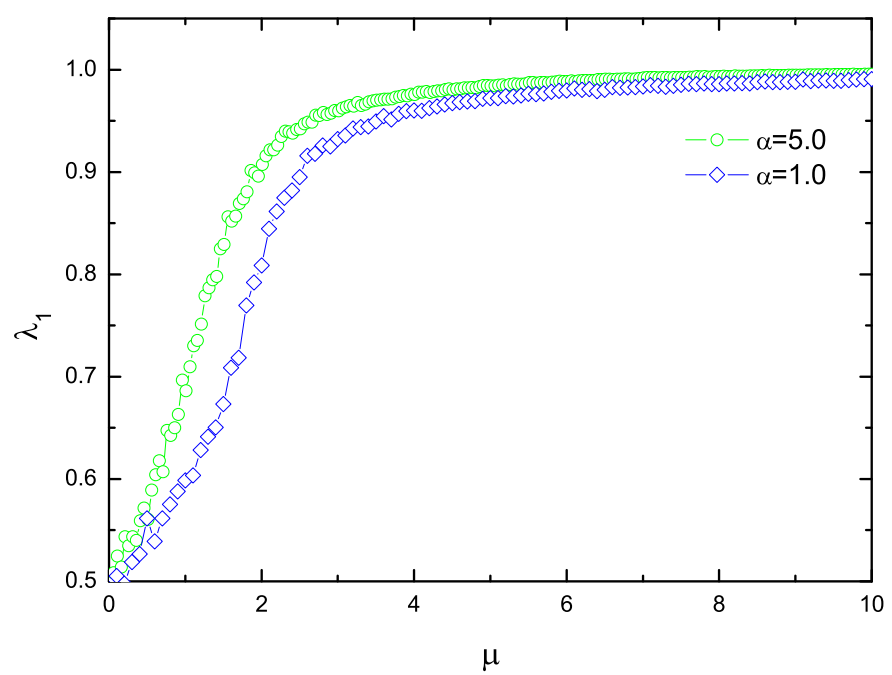

Figure 10: Parameter $\lambda_{1}$ in the final stationary state as a function of parameter $\mu$ for different $\alpha$.

are almost consistent. It has only some effects on the accurate of simulation. Second, When $\gamma<1.0$ in $\gamma$-mechanism for technical progress, the productivity declines with the time evolution. Then the system could not reach the state of specialization. This result is also meaningful for real economic system.

\section{Concluding Remarks}

A distinctive feature of the organization of a human society is the division of labor. From the classical economic theory[11], the division of labor comes from the development of endogenous comparative advantages. So there is the intrinsic relationship between technical progress and the evolution of the division of labor. But how and why it emerges from the system consists of identical individuals? We have studied the formation of labor division by the approach of statistical physics. The results reveal that there is a phase transition with this pattern formation. Although the progress of productivity dominated the phase transition occurs or not, the competitive cooperation among the agents has important effects on the critical point. So the market formation and labor division are usually reinforced each other. All the above results give us deep understanding to the evolution of labor division.

Studying the economy as an evolving complex system, we can avoid the standard economic assumptions of equilibrium based on rational behavior by agents. And the concepts and methods developed in statistical physics is helpful to uncover fundamental 


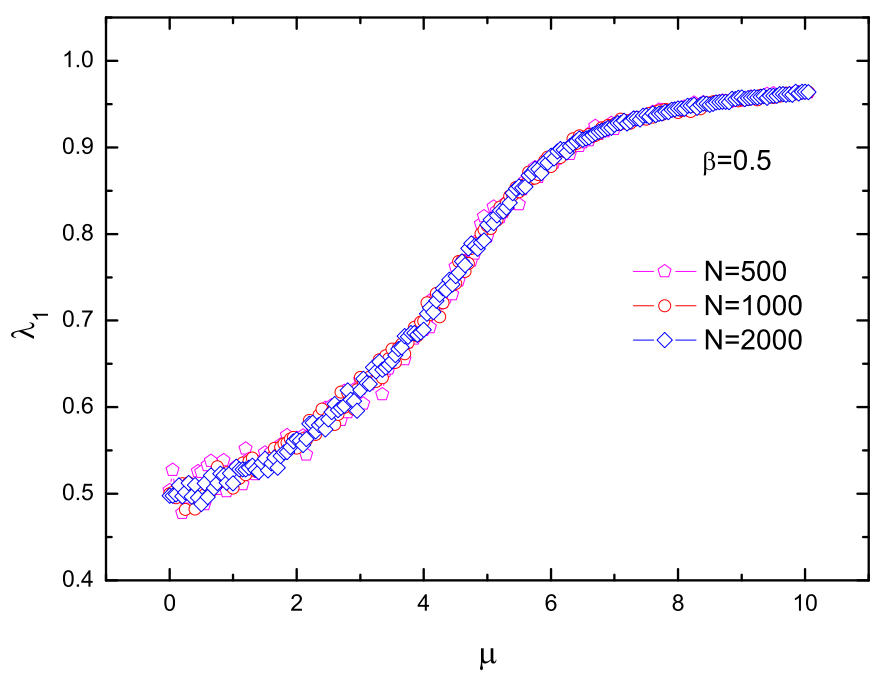

Figure 11: When $\beta=0.5, \alpha=1.0, \lambda_{1}-\mu$ curves for different population size $N$. The curves are almost consistent.

principles governing the evolution of complex adaptive systems. So the approaches presented here have potential applications in a variety of economical, biological and financial problems.

\section{Acknowledgement}

This work was supported by the National Science Foundation under Grant No.10175008 and National 973 Program under Grant No.G2000077307. We also thanks to all members of Professor Yang's Group and faculties from Department of System Science for their warm discussion.

\section{References}

[1] R.N. Mantegna, H.E. Stanley, Scaling behavior in the dynamics of an economic index, Nature 376 (1995) 46-49.

[2] LA,N, Amaral, P. Gopikrishnan, V. Plerou, H.E.Stanly, A model for the growth dynamics of economic organizations, Physica A 299(2001) 127-136.

[3] A. Ponzi, Y. Aizawa, Evolutionary financial market models, Physica A 287(2000) 507-523. 
[4] V. Plerou, P. Gopikrishnan, L.A.N. Amaral, M. Meyer, H.E. Stanley, Scaling of the distribution of price fluctuations of individual companies, Phys. Rev. E 60 (1999) 6519-6529.

[5] N.A. Criss, Review of Mantegna and Stanley, an introduction to econophysics, Physics Today53(2000)12.

[6] D. Challet, Y.-C. Zhang, Emergence of cooperation and organization in an evolutionary game, Physica A 246(1997) 407.

[7] D. Challet, M. Marsili, Phase transition and symmetry braking in the minority game, Phys. Rev. E 60 (1999)6271.

[8] M. Marsili, Market mechanism and expactations in minority and majority games, Physica A 299(2001) 93-103.

[9] Krzysztof Kacperski, Janusz A. Holyst Phase transitions as a persistent feature of groups with leaders in models of opinion formation, Physica A 287 (2000) 631-643.

[10] J. O. Kephart, T. Hagg, and B. A. Huberman, Dynamics of computational ecosystems, Phys. Rev. A 40, 404-421(1989); Collective behavior of predictive agents, Physica D 106, 48-65(1990).

[11] X. Yang, Y.-K. Ng, Specialization and economic organization-a new classical microeconomic framwork, North-Holland, 1993.

[12] K. Binder, D.W. Heermann, Monte Calo Simulation in Statistacal Physics, Springer-Verlag, 1988.

[13] Jian-Yang Zhu, Z. R. Yang, Exact solution of the kinetic gaussian model, Phys. Rew. E59, (1999)1551.

[14] K. J. Arrow, The economic implications of learning by doing, Review of Economic Studies 29, 155-173(1962) 